\title{
IMPROVING PORE PERFORMANCE
}

"It'll never work" is a comment all inventors contend with. Innovators from the Wright brothers to the inventors of the scanning tunnelling microscope, Gerd Binnig and the late Heinrich Rohrer, faced such scepticism. So did David Deamer and Dan Branton when they suggested in the mid-1990s that DNA might be sequenced by pulling single strands through nanopores and deducing the nucleotides from their distinct blocking effects on ion currents through the pore. Some geneticists dismissed the idea as crazy.

Now at least one commercial company - Oxford Nanopore, drawing on the work of Hagan Bayley - is developing nanopore technology for commercial molecular-sensing devices including gene sequencing; a European project called nanoDNAsequencing is pursuing the idea; and the NIH has recently awarded nearly $\$ 17$ million to eight teams working on nanopore sequencing.

Some of these efforts use pores carved into inorganic thin films such as silicon, and others (like Oxford Nanopore) use the natural membraneprotein nanopore $\alpha$-hemolysin. But there is a common view that graphene could be a game-changer. Graphene's intrinsic electrical conductivity might provide a built-in readout mechanism, and its single-atom thickness - which should mean that only a single base pair of threaded DNA blocks the pore at any moment - could give a high-resolution readout.

Branton's group ${ }^{1}$ and others ${ }^{2,3}$ demonstrated the blocking of ion flow through a graphene nanopore by DNA in 2010 , albeit without base discrimination. One of the most promising ideas is to use currents in graphene nanoribbons, localized around the edges by their specific atomic-scale structure, to sense the identity of the base occluding a hydrogen-passivated central pore. Here the pore does little to diminish the ribbon's overall conductivity, but the threaded DNA modulates the current, hopefully in a base-specific way. This principle is supported by theoretical studies ${ }^{4}$, but would be complicated in reality by the screening effects of ions in the electrolyte solution, and by the need for atomic-scale precision in defining the ribbon edges.

Now Girdhar et al. suggest that a graphene nanopore sensor like this might work best if its electronic properties are brought under active control, in particular by introducing a gate electrode to adjust the carrier concentration in the graphene sheet ${ }^{5}$. This would make the device a kind of transistor. Girdhar et al. posit a structure in which a single graphene ribbon connected to source and drain contacts is sandwiched between oxide thin films (also pierced by coaxial nanopores) to insulate it from the solution, while a back gate under the bottom oxide layer controls the carrier concentration.

The calculations show there are resonances and other nonlinearities in the transmission of the nanoribbons as a function of carrier energy, and that the width of the ribbon and pore, and the precise edge structure, offer

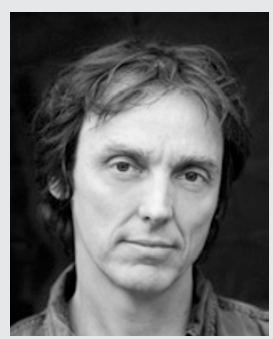

PHILIP BALL

several parameters for tuning the device sensitivity.

Under the right conditions, translocation of the DNA can be watched step by step as the screw-like 'rotation' of the double helix produces changes in the sensing current.

Getting base discrimination which these calculations don't yet exhibit - would involve careful control of the DNA motion, so that the strand doesn't move too fast (or reverse direction) and doesn't wander off-centre and get abraded by the pore edge. This could be achieved by adding additional graphene layers to control the speed and focus the position in the pore, as suggested here and elsewhere ${ }^{6}$. Work in progress, then - but the path is coming clearer.

References

1. Garaj, S. et al. Nature 467, 190-193 (2010).

2. Merchant, C. A. et al. Nano Lett. 10, 2915-2921 (2010).

3. Schneider, G. F. et al. Nano Lett. 10, 3163-3167 (2010).

4. Saha, K. K., Drndic, M. \& Nikolic, B. K. Nano Lett. 12, 50-55 (2012).

5. Girdhar, A., Sathe, C., Shulten, K. \& Leburton, J.-P. Proc. Natl Acad. Sci. USA http://dx.doi.org/10.1073/ pnas. 1308885110 (2013).

6. Avdoshenko, S. M. et al. Nano Lett. 13, 1969-1976 (2013).

Advances in photochemistry have profoundly impacted the way in which biology is studied. Now, a photoactivated enzymatic patterning method that offers spatiotemporal control over the presentation of bioactive proteins to direct cells in three-dimensional culture significantly expands the available chemical toolbox.

\section{Daniel L. Alge and Kristi S. Anseth}

ells in tissues are embedded within the extracellular matrix (ECM) - a complex milieu that varies from tissue to tissue. Far from being a static collection of biological macromolecules, the ECM is an assembly of biochemical and biophysical cues that are presented to cells in a dynamic fashion and affect cellular organization and function. Indeed, the dynamics of the ECM are critical to development, tissue healing and many diseases. In vitro systems capable of 